\title{
THE IRRIGATION TREATMENT FOR PAINFUL SHOUĹDERS.
}

\section{By ROBERT LEE PATTERSON, Jr., M.D. and PHILIP D. WILSON, M.D., New York, N.Y.}

From a review of the cases seen in our clinics at the Hospital for the Relief of the Ruptured and Crippled, it is evident that bursitis of the shoulder is the diagnosis now most often given to painful shoulders. From I936 to I940 it was recorded in 343 cases. Most of the shoulders formerly listed as periarticular inflammation, arthritis, neuritis, radiculitis, and myositis are now recognized as bursitis either with or without calcification (Patterson, R. L., Jnr. and Patterson, R. H., I940). This more frequent diagnosis has been made possible through increasingly careful clinical examinations, through improved $\mathrm{X}$-ray technique (Figs. I, A, B \& C, Art Plate) and through a greater understanding of the underlying pathology.

Out of 450 cases of painful shoulder seen during the past four years the majority were sent for physical therapy in the form of baking and diathermy. However, I63 were irrigated according to the method originally described in 1937 by one of the authors (R. L. P., Jr., I937). On the basis of our results in these cases we are convinced that irrigation is indicated for certain types of painful shoulder and we can recommend its use in a large proportion of cases.

\section{Causes :}

In order to understand the rationale behind the irrigation treatment, it is advisable to consider briefly the various conditions which produce bursitis and painful shoulder. We will not discuss bursitis due to bacterial infection here as it is extremely rare and only occurred in one case out of our series of $45^{\circ}$.

Anything that injures the bursal sac and disturbs its function will give symptoms of bursitis. Repeated friction from calcium deposits in the underlying tendons seems to be the most common cause. In one group of 77 cases, 55 were caused in this manner. We know that the deposit tends to rupture eventually into the sac and to produce a chemical irritation of its walls (Fig. 2, Art Plate). If absorption of the deposit or bursal contents does not take place within two or three weeks, symptoms persist and relief can be offered by drainage of the bursa through irrigation. Moreover, if the deposits remain in the tendons and capsular linings and cannot work themselves out, they continue to act as irritants. Needling of these deposits in order to aspirate them or to make them rupture into the bursa will be beneficial.

Repeated irritation from arthritic changes in the under surface of the acromion, in the acromio-clavicular joint, and in the tuberosity of the humerus accounts for many painful shoulders. In these cases our aim in irrigating is to relieve the distension of the bursa, produced by the irritation.

Tension in the bursa may also be caused by hæmorrhage into the sac as a result of contusions about the shoulder or falls on the arm, often accompanying a Colles fracture of the wrist. This type of hæmorrhagic bursitis is the result of a pinching of the bursa between the acromion and the head of the humerus. If symptoms persist for more than forty-eight to sixty hours, the tension can be relieved by irrigation. 


\section{Types :}

We have divided our cases of painful shoulder into four types according to their response to treatment.

I. Those relieved immediately.

2. Those relieved, but not dramatically.

3. Those relieved temporarily.

4. Those not relieved. In these irrigation is contraindicated.

Characteristic of the first type are the patients who have had acute pain in the shoulder for less than ten days, who on examination show marked pain, slight swelling, and localized tenderness about the head of the humerus, pain on attempted motions of the arm, and whose X-rays reveal a soft, diffuse, not too large calcified shadow in the region of the bursa. Irrigation will give these patients almost immediate relief and will bring a return to normal function within a few days (usually 4 to 5 days) (Figs. 3 A \& B, Art Plate). Also in this group are cases with acute pain due to hæmorrhage. Their response to irrigation is equally dramatic.

The second group consists of cases who have had a dull ache in the shoulder or region of the deltoid attachment into the humerus off and on for weeks, months, or even years, whose discomfort is gradually increasing, is aggravated by use, and interferes with activity. These patients show on physical examination a slight limitation of complete abduction because of pain ; they have soreness about the shoulder but no atrophy. Their X-rays reveal a small, hard, smooth, calcified deposit or deposits usually in a tendon about the shoulder. Often they have a definite increase in bursal fluid. Irrigation of these shoulders is advised, attempt being made to strike the calcium deposit. Although the removal of that factor as an irritant does not cure the patient, it hastens the healing of the underlying tendinitis. Following irrigation, the arm is sore, motions are painful and limited, and sedatives are required for twenty-four to forty-eight hours. A sling is used for a week and the patient is urged not to move the arm. An electric pad or infra-red rays' should be the only treatment. As the soreness slowly subsides the sling is removed and the patient allowed to begin to use the extremity. If he sleeps at night without discomfort, slow pendulum exercises can be started. In none of our cases have we seen a shoulder remain "frozen" after the process has quietened down, when exercises have been carried out faithfully. It must be emphasized that these patients do not recover until the underlying disease is arrested and full painless motion is attained (Figs. $4 \mathrm{~A} \& \mathrm{~B}$, Art Plate). The return of full motion usually takes from four to ten weeks, which seems an exceedingly long time in an otherwise healthy person. The patient is apt to be discouraged and it is helpful to explain to him at the outset what he should expect, both as to pain and time.

The third type of painful shoulder is that in which irrigation may provide only temporary relief and may, in many instances, have to be repeated. As we have said, when the tension in the bursa is due to irritation from bony changes about the joint (Figs. I C \& 5, Art Plate) which we call arthritis, relief of the tension gives real comfort to the patient. When the bursa becomes inflamed again because of increased activity of the arm, irrigation is again necessary. Often the time interval between irrigations is several months. Three of our cases were asymptomatic for as long as seven months, six months, and five months, respectively. Upon re-irrigation, two are still without pain after one year, and the third has 
PLATE I.
Irrigation Treatment for Painful Shoulders - R. L. Patterson and P. D. Wilson

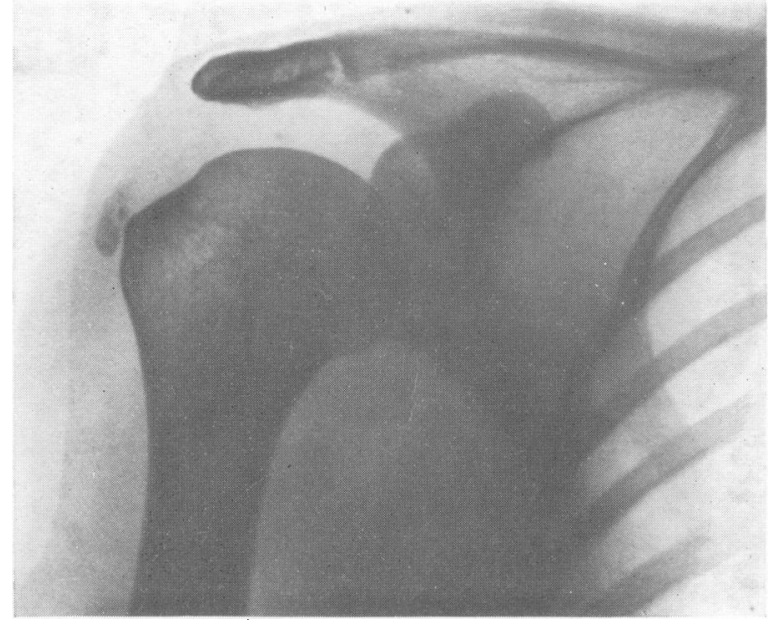

FIG. IA.-Calcium deposit is easily seen with arm in external rotation.

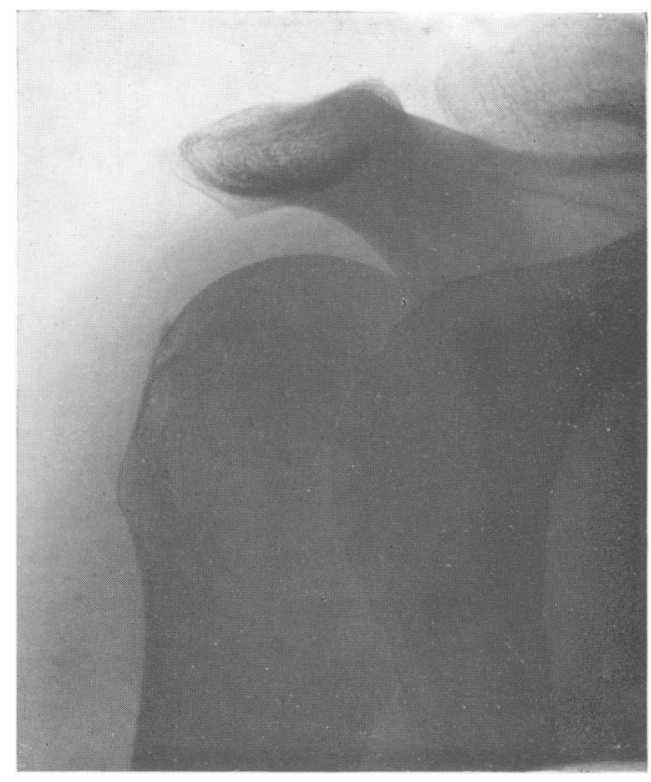

FIG. IB.-Same as Fig. IA with arm in internal

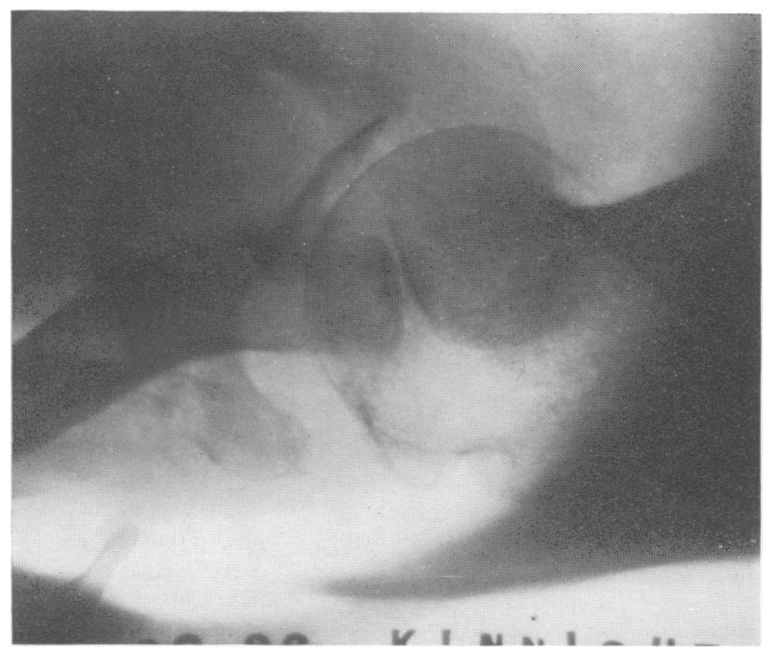

FIG. IC.-Special axillary view which aids in diagnosis of shoulder lesions: rotation. Deposit barely visible.

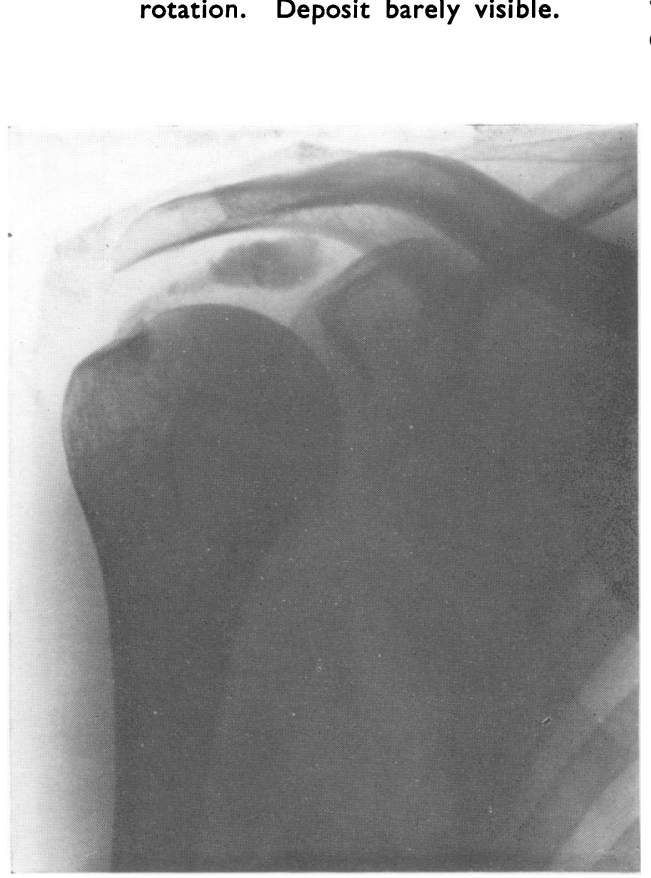

FIG. 2.-Calcification rupturing from tendon into bursa. Acute symptoms of only two days. 
PLATE II.

Irrigation Treatment for Painful Shoulders - R. L. Patterson and P. D. Wilson
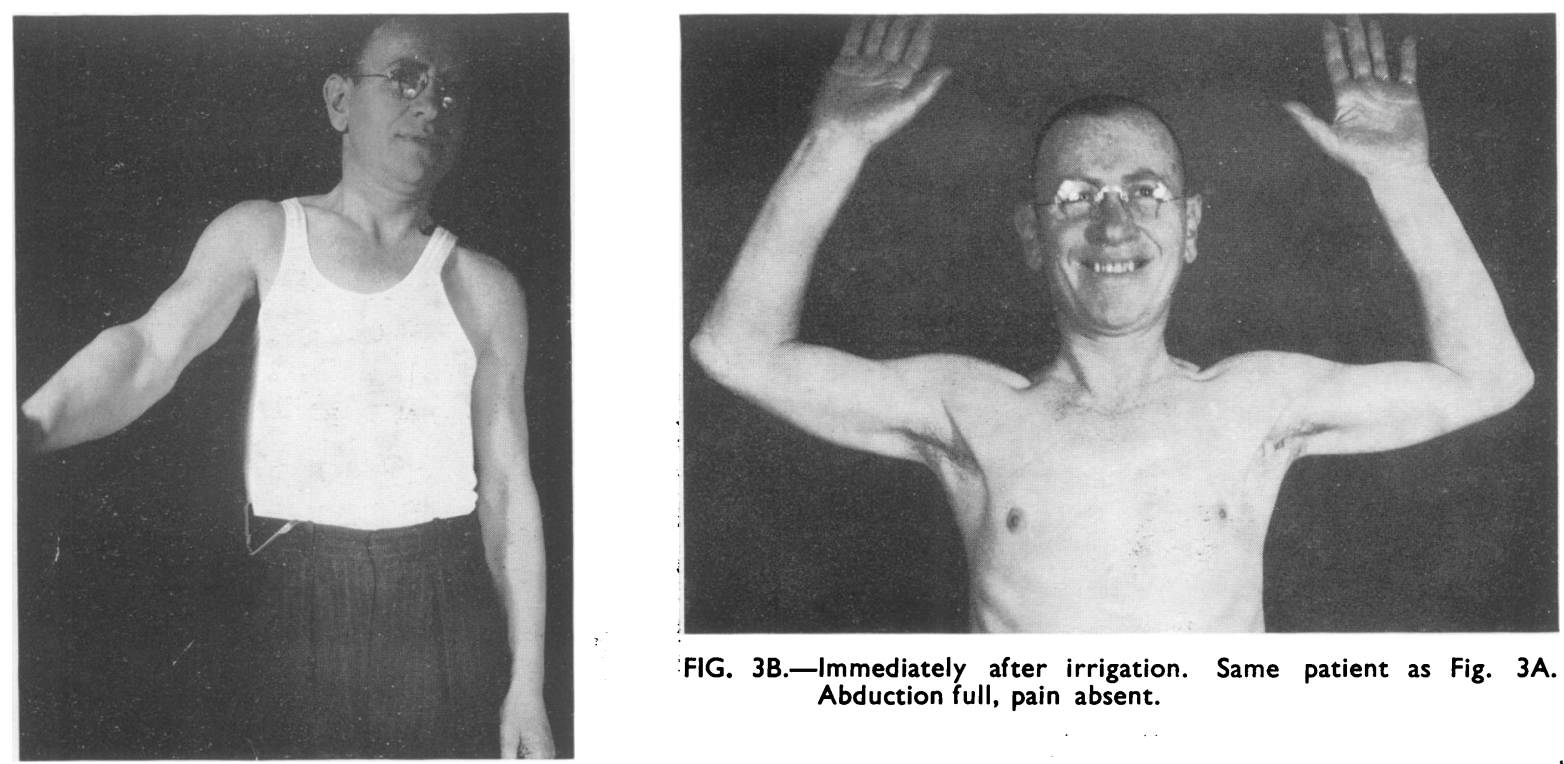

FIG. 3B.-Immediately after irrigation. Same patient as Fig. 3A. Abduction full, pain absent.

FIG. 3A.-Before irrigation: abduction 35 degrees, pain marked for 24 hours.
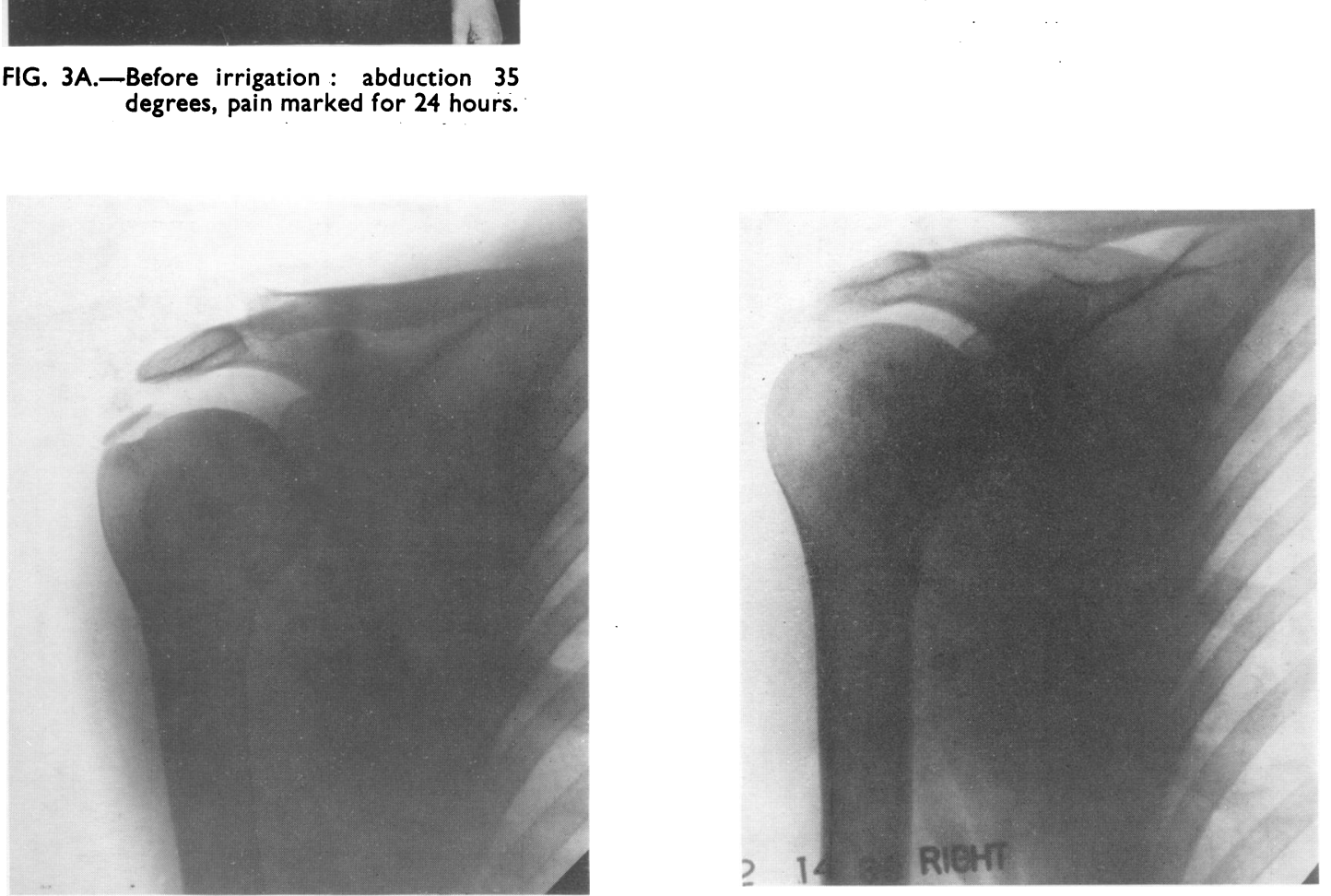

FIG. 4A.-Calcium deposit producing symptoms for seven years. Removal by open operation was performed.

FIG. 4B. Same patient as Fig. 4A. Taken one year after operation. Symptoms still present. 


\section{Irrigation Treatment for Painful Shoulders - R. L. Patterson and P. D. Wilson}

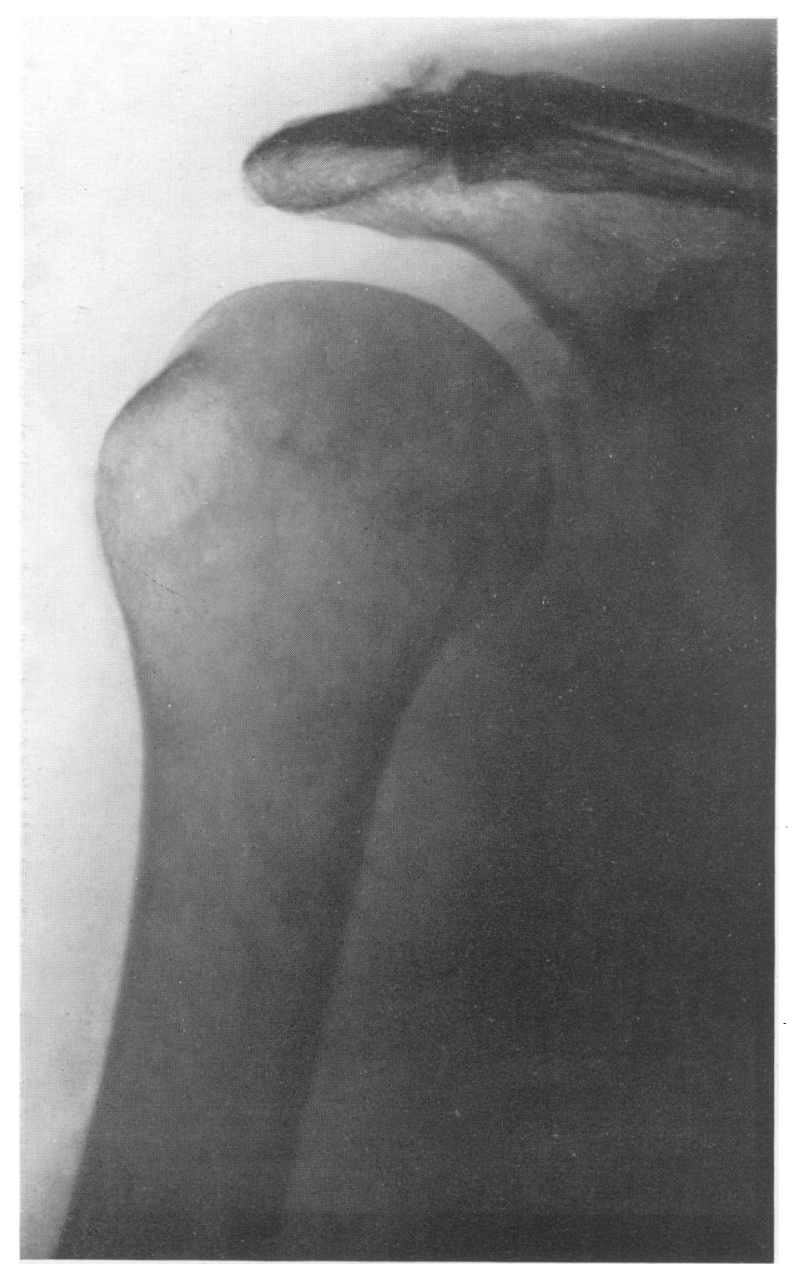

FIG. 5.-Arthritis of shoulder with calcified deposits in acromio clavicular joint.

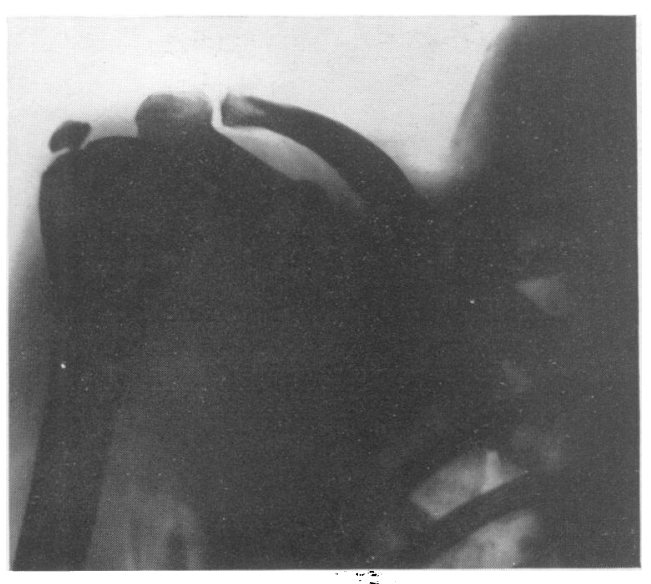

FIG. 6.-Four cc. of calcium obtained from this large deposit by irrigation. $X$-rays after irrigation, however, showed the appearance unchanged. Later required open removal.

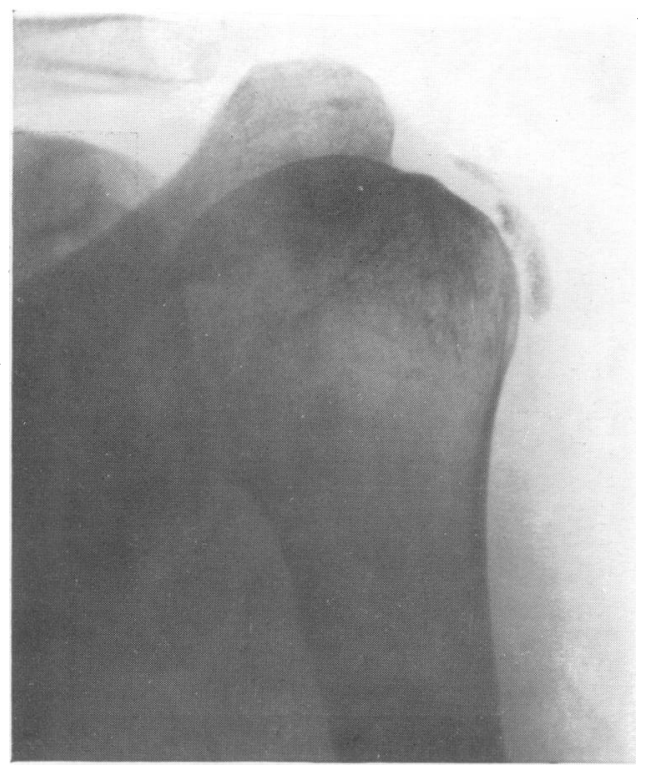

FIG. 7.-Multiple deposits found in supraspinatus and infraspinatus tendons at open operation. 
FIG. 8.-Needles in correct position to irrigate right subacromial bursa.

FIG. 9.-Position of needles in calcium deposit.

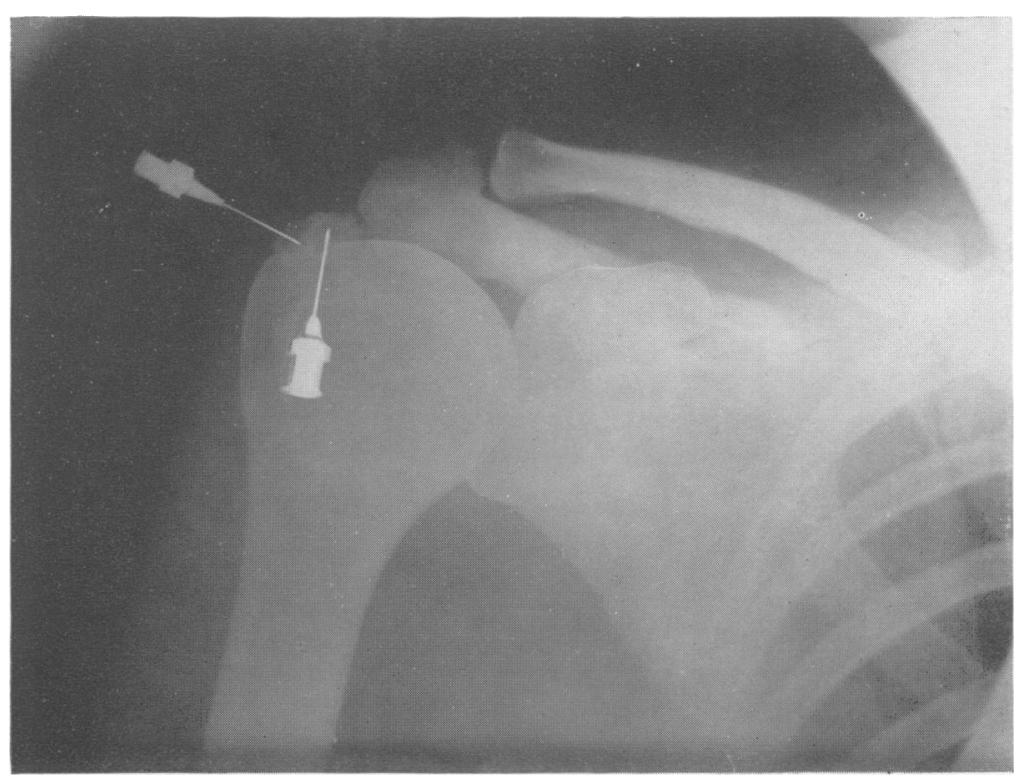


only occasional slight discomfort. In these there was no gross evidence of calcium in the washings or by X-ray. A thick tenacious, yellowish material like ganglionic fluid was obtained, however, which by chemical analysis showed a positive test for calcium.

Let us now discuss the types of shoulder which fail to respond satisfactorily to irrigation. About twenty-nine per cent. of the cases of painful shoulder studied were diagnosed as periarticular inflammation, a condition which according to our findings was second only in number to bursitis. Irrigation in these cases does not give the expected quick relief of pain and the convalescence is long. We consider that the diagnosis of periarticular inflammation indicates tendinitis, capsulitis, or myositis, that the condition is chronic, and that the shoulder is probably only a local manifestation of some systemic infection or metabolic disturbance such as gout, toxemia, or arthritis. Accordingly, irrigation of the local condition proves of little value. Attempt should be made to eliminate any focus of infection that may be present. Dickson and. Crosley (I932), found seventy-eight per cent. of two hundred cases to have a focus in the teeth, tonsils, sinus, or genito-urinary tract. In thirty-five cases of periarticular inflammation irrigated by us, no calcified material was obtained, the patients suffered an extreme degree of discomfort afterwards, and the shoulder slowly became stiff. Four weeks after the procedure, only two cases had more than fifteen degrees of abduction and none had any external rotation. As motion became more and more restricted, pain subsided. At ten weeks following irrigation, most of the patients complained of limited motion only. Exercises were carried out. The average case required three to eight months before a full range of motion was obtained, but in none of the cases did function fail to return, though in some it took as long as a year. Since the above course is characteristic of this type of painful shoulder regardless of the treatment used, irrigation cannot be recommended.

Irrigation is also contra-indicated when subjective symptoms of numbness and pain are present without any positive objective findings. Such symptoms should lead one to suspect a diagnosis of early cervical arthritis, cervical rib, or scalenus anticus syndrome.

When on X-ray examination of the shoulder we find a very large calcareous deposit (Fig. 6, Art Plate) or one divided into several small deposits (Fig. 7, Art Plate) which seem to be in various locations in the tendon or capsule, irrigation is not advisable. It is often impossible to strike each of the deposits or to remove the entire contents of the large depositions, since the flakes are infiltrated into the tendon fibres and the walls of the bursa. If it seems that more than one needling will be necessary it is better to perform an open operation on the shoulder and take out all the deposit at once. It is discouraging to a patient if after irrigation $\mathrm{X}$-rays still show calcium.

Irrigation, lastly, is contra-indicated when $\mathrm{X}$-rays reveal suspicious lesions which might denote tuberculosis, syphilis, or infectious arthritis.

For a chronically frozen shoulder, irrigation of the bursa followed by an infiltration of normal salt solution into the soft parts of the shoulder and stretching or manipulation, has been carried out in several cases with satisfactory results. With the patient under anæsthesia, two needles are inserted into the bursa and if possible all the calcium is removed. In many cases no calcium can be demonstrated previously by X-ray, so in these the bursa is simply washed out with 
saline. Next, one of the needles is withdrawn and about Ioo to 200 cubic centimetres of the salt solution are injected in to the remaining needle. Then this needle is removed and the shoulder manipulated. We believe that irrigation dilutes hæmorrhage, helps to prevent a reformation of adhesions in and about the bursa, and lessens the severe pain following the usual manipulation.

\section{Technique :}

The equipment necessary for irrigation consists of one hypodermic needle, 50 cubic centimetres of I per cent. novocaine without adrenalin, a 20 c.c. syringe, a sharp pointed scalpel, two I8 gauge needles, two and one-half inches long, and Ioo c.c. normal saline solution.

The patient is placed face upwards on a table, care being taken not to move, twist, or touch the arm involved. A small pillow under the head and another under the elbow help to make the position more comfortable. Definite spots of tenderness may be marked with an aniline dye such as brilliant green, which remains visible even after the application of iodine. Next, the neck, shoulder, and upper arm to the region of the deltoid attachment into the humerus are prepared with iodine and alcohol. With a hypodermic needle a small amount of I per cent. novocaine solution is then injected into the skin over the anterior aspect of the bursa about two fingers' breadth lateral to the coracoid process of the scapula. Then one of the I8 gauge needles is inserted here. If the whole shoulder is acutely painful, the skin should first be cut with the tip of a scalpel to avoid the pressure on inserting the needle. Considerable discomfort can also be eliminated by waiting five minutes after using the novocaine before putting in the larger needle. In directing the needle the head of the humerus is often touched, as the distance between the skin and the bone is only about one-half an inch. If this is done the needle should be withdrawn about one-eighth of an inch and the tip of the needle will be in the bursa. The second needle is inserted into the region of the superior facet of the greater tuberosity of the humerus where the supraspinatus tendon is attached. This spot which usually corresponds with the point of maximum tenderness, is to be found about one finger's breadth below the tip of the acromion, since the patient holds the whole extremity adducted and internally rotated (Fig. 8, Art Plate). As each needle is inserted a small amount of novocaine is put in and an attempt is made to aspirate any material encountered. If the needle appears to be stopped up by the calcified material, a few cubic centimetres of novocaine forced through will dilute the material sufficiently to aspirate. The position of each needle should be slowly changed as the novocaine is inserted until the fluid injected through one needle gushes out the other showing that both are in the bursal sac (Fig. 9, Art Plate). After the flow is well established with novocaine, normal saline is then used. If no calcium is seen in the washing the X-rays are again consulted and an attempt is made to locate it with one of the needles. If the calcium has not ruptured into the bursa it must be sought in the tendons about the shoulder. Considerable patience is often required in finding the deposit, but with care it can be done without causing the patient harm or pain. We have not found any deposit on needling or even at open operation which was of such density that at least a part of it could not be removed by irrigation. However, if the tendon is badly involved, the entire removal by irrigation is usually impossible. If only one needle enters the deposit, as demonstrated by seeing calcium flakes in the fluid, the point of the other needle is put in the same region so that a flow can be established and the material washed out of the tendon. The process should be performed slowly since a sudden distension of the tendon produces con- 
siderable pain. When the washings are fairly clean, both needles are removed and we ascertain if any tender spots are still present. If a local point of tenderness is found, the needle is re-inserted directly into it and novocaine injected. Following the removal of the needles, the patient should have no pain on passive abduction and external rotation of the arm. The patient is then given a sling and a prescription for sedatives. We routinely recommend that the patient who has had extreme pain and several days of disability with little or no sleep, remain in the hospital and that if he should have increasing discomfort after the anæsthesia has worn off, he be given morphine. The use of the extremity is not permitted until the patient has a fair degree of painless motion. This may take from three to fourteen days, averaging about five days in the acute cases.

We do not recommend fluoroscopic examination as an, aid to irrigation for three reasons: (I) It is extremely difficult to keep the field sterile; (2) The whole shoulder area is hard to visualize and after the infiltration of a few cubic centi-c metres of novocaine, the calcium deposit often cannot be identified; (3) The exposure to the shoulder of the patient and to the hands of the surgeon is dangerous.

\section{Results :}

Out of I68 cases of painful shoulder treated by the irrigation method during the past four years, we have been able to determine the results on II8. Seventythree of these we graded as good and 45 as poor. At first glance this percentage (6I per cent.) of good results seems rather low and for the whole group of cases treated is actually unsatisfactory. However, an analysis of the poor results shows that if irrigation had been performed only in the types of cases for which we have recommended it, the percentage of good results would rise from 6I per cent. to 90 per cent. Thirty-five cases out of the forty-five graded as poor proved, upon further study, to belong to the group called periarticular inflammation or periarthritis. We have already discussed this condition and have tried to show why irrigation failed to cure it. The diagnosis of periarticular inflammation was known in twenty out of the thirty-five cases; nevertheless, it was thought advisable to irrigate, as at that time the treatment was first being used and no positive facts were available as to the type of case for which it was best suited. However, in the fifteen other cases the diagnosis was mistaken and only made after further study and care.

There has been only one recurrence when irrigation was performed during an initial acute attack, when calcium was obtained, and when relief was immediate. In this patient the calcium was removed in the original irrigation as proved by an $\mathrm{X}$-ray taken after the procedure and after being free of symptoms for four years, she again had acute pain and new X-rays showed calcium deposition. She was re-irrigated, calcium was again obtained and she had relief from pain in twentyfour hours. She returned to her work in five days with full use of the extremity.

In nine cases out of the forty-five failures, open operation was subsequently performed. It is worth while to discuss briefly the findings at operation. In four cases out of this group no calcium had been obtained on irrigation, pain from distension of the tendon was extreme following the procedure and no improvement was noticed after forty-eight hours. Each was operated on. In three of them the bursal wall was found to be thickened, inflamed, and reddened. No free fluid was present. Puncture wounds were seen in the walls of the sac but the deposit was still lying untouched in the tendons. In the fourth of this group the needle 
had entered the distal end of the deposit and some of the material was exuding into the bursa but the main part of the deposit was still present in the tendon. We believe that there was a chance that this case might have been cured if he had had patience to wait for several weeks.

In four other cases out of the nine requiring open operation, the deposit was too large to be removed completely. In each the deposit was entered with the needles and as much as four cubic centimetres of the material removed, though subsequent X-rays showed shadows still present (Fig. 6). On incising the bursal walls some calcareous material was found in the cavity, some free fluid was seen but what was most remarkable was an infiltration of the lining membrane with small flakes of the calcified material. Deposits were also found in the tendons, though they were small.

The ninth and last case in this group of failures, was that of a young woman of twenty-six years who had a dull ache in the shoulder and arm. All motions were free but at night and after exercise pain was disturbing. X-rays were reported on three occasions as negative. Irrigation of the bursa was done with relief for eleven weeks, repeated with relief for eight weeks, and repeated once more with no relief. At operation, two cubic centimetres of white milk-like material were found lying free in the bursa. The walls were not very thick and there was no deposit in the tendons. Her convalescence was short and for three and a half years she has been asymptomatic.

In these nine operative cases the average length of hospitalization was about six days. There was an average lapse of four months before pain had disappeared and a full range of motion was obtained.

\section{SUMMARY.}

(I) Out of 450 cases of painful shoulder studied, 343 were given a diagnosis of bursitis, either with or without calcification.

(2) The rationale of irrigation is discussed on the basis of the most common causes of bursitis and painful shoulder.

(3) The types of cases which do and those which do not respond to irrigation are described.

(4) The technique of irrigating painful shoulders with novocaine and saline is described.

(5) I68 cases were irrigated. Out of the II8 cases on which results were available, 73 were graded as good and 45 as poor.

(6) An explanation is given for the poor results and an attempt is made to show that if irrigation is properly employed, about 90 per cent. good results can be expected.

\section{REFERENCES.}

PATTERSON, R. L. Jr., and PATTERSON, R. H. (1940). Further observations on the treatment of bursitis of the shoulder. The American Journal of Surgery, 49, 403.

PATTERSON, R. L. Jr., and DARRACH, W. (1937). Treatment of acute bursitis by needle irrigation. The Journal of Bone and Joint Surgery, 19, 993,

DICKSON, J. H., and CROSLEY, E. H. (1932). Periarthritis of the shoulder: an analysis of 200 cases. The Journal of the American Medical Association, $99,2252$. 